\title{
Cognitive behavioural therapy for insomnia for patients with co-morbid generalized anxiety disorder: an open trial on clinical outcomes and putative mechanisms
}

\author{
Markus Jansson-Fröjmark ${ }^{\star}$ (iD and Kalle Jacobson \\ Centre for Psychiatry Research, Department of Clinical Neuroscience, Karolinska Institute, and Stockholm Health Care \\ Services, Region Stockholm, Sweden \\ ${ }^{\star}$ Corresponding author. Email: markus.jansson-frojmark@ki.se
}

(Received 10 February 2020; revised 9 December 2020; accepted 9 December 2020; first published online 28 January 2021)

\begin{abstract}
Background: Very little is known concerning the efficacy of psychosocial treatments for patients with insomnia disorder co-morbid with generalized anxiety disorder (GAD).

Aim: The aim was to examine the efficacy of cognitive behavioral therapy for insomnia (CBT-I) for patients with insomnia disorder co-morbid with GAD.

Method: Given the limited, previous research on therapies for patients with insomnia disorder co-morbid with GAD, an open trial design was used. Twenty-four patients with insomnia disorder and GAD were administered CBT-I across 10 weeks. Across the study period to 6 months follow-up, the participants completed measures indexing insomnia, anxiety, worry, depression, functional impairment, quality of life, treatment perception (credibility, expectancy and satisfaction), adverse events and putative mechanisms.

Results: Moderate to large effect sizes for CBT-I were observed for insomnia symptoms. In terms of insomnia severity, approximately $61 \%$ of the patients responded to CBT-I and $26-48 \%$ remitted. Moderate to large effect sizes were also demonstrated for GAD symptoms, depression, functional impairment and quality of life. Roughly one-third of the participants reported an adverse event during CBT-I. Five of the seven putative mechanisms were significantly reversed in the expected direction, i.e. all four cognitive process measures and time in bed.

Conclusions: This open trial indicates that CBT-I is an efficacious intervention for patients with insomnia disorder co-morbid with GAD. The results highlight the need for further research using a randomized controlled trial design with analyses of mechanisms of change.
\end{abstract}

Keywords: cognitive behaviour therapy; generalized anxiety disorder; insomnia

\section{Introduction}

Insomnia disorder is a sleep condition that means difficulties in falling asleep, maintaining sleep, or waking up too early in the morning and associated daytime impairment (American Psychiatric Association, 2013). Insomnia disorder is a prevalent condition, affecting approximately $10 \%$ of the general population (Morin et al., 2015), and is often co-morbid with psychiatric diagnoses (Ford and Kamerow, 1989; Kim et al., 2012), particularly so with anxiety and mood disorders. When insomnia is co-morbid, it has been related to more severe psychiatric symptomatology (McCall et al., 2010; Sunderajan et al., 2010; Taylor et al., 2005) and higher risk for new psychiatric episodes (Sylvia et al., 2012; Troxel et al., 2012). As co-morbid insomnia is regarded as an independent health problem that warrants treatment in its own right (American Psychiatric 
Association, 2013), research attempts have been made during the past decade to explore the efficacy of various treatments for co-morbid insomnia.

Cognitive behavioural therapy for insomnia (CBT-I) is a multi-component and evidence-based treatment (Morin et al., 2015). CBT-I aims to alter sleep-related misconceptions and thought patterns, to change maladaptive sleep habits, and to lower sleep-disrupting arousal. Recent guidelines have underscored that CBT-I should be considered the treatment of first choice for patients with insomnia disorder, either delivered face-to-face or via the internet (Qaseem et al., 2016; Riemann et al., 2017; Zachariae et al., 2016). Also, several meta-analyses and reviews have demonstrated the efficacy of CBT-I in the context of other mental disorders, with effect sizes for key insomnia symptoms ranging from 0.68 to 0.91 (Geiger-Brown et al., 2015; Jansson-Fröjmark and Norell-Clarke, 2016; Wu et al., 2015). However, relatively few studies have been carried out exploring CBT-I for insomnia co-morbid with other mental disorders, and there has been a limited number of mental disorders included in trials, predominantly insomnia co-morbid with alcohol dependence, post-traumatic stress disorder (PTSD) and major depression (Geiger-Brown et al., 2015; Wu et al., 2015).

One of the many psychiatric co-morbidities that has received little attention in treatment studies is generalized anxiety disorder (GAD). There are several reasons why treatment research is needed for those with insomnia disorder co-morbid with GAD. First, the sleep difficulties among GAD patients are characteristic insomnia symptoms, including difficulty falling and staying asleep, as well as restless sleep (American Psychiatric Association, 2013). Second, insomnia disorder and GAD very often co-occur. Among adult patient samples, $85-90 \%$ of GAD patients report dissatisfaction with their sleep, and $52-68 \%$ can be classified as having moderate or severe insomnia (Bélanger et al., 2004; Brenes et al., 2009; Ferre Navarrete et al., 2017). Using structured interviews for the assessment of insomnia disorder and GAD, 9-13\% of insomnia participants meet criteria for GAD (Breslau et al., 1996; Mellinger et al., 1985). Polysomnographic findings also suggest that GAD patients, relative to individuals without $\mathrm{GAD}$, in objective terms display increased wake time as well as decreased sleep efficiency and total sleep time (Monti and Monti, 2000).

Two additional reasons should be pointed out as to why clinical research is warranted for those with insomnia disorder co-morbid with GAD. Research shows that, among GAD patients, worry and poor sleep interact over time. In one study using ecological momentary assessment, worry and sleep quality formed a bidirectional relationship across one week (Thielsch et al., 2015). In another study, it was more common for anxiety disorders to develop before insomnia disorder when co-morbid cases were investigated (Johnson et al., 2006). At the same time, other investigations have reported results consistent with the notion that insomnia might increase the risk of anxiety (Breslau et al., 1996; Gehrman et al., 2013; Jansson-Fröjmark and Lindblom, 2008; Neckelmann et al., 2007; Sivertsen et al., 2014). Furthermore, examining the aetiologicol overlap between the genetic and environmental influences on insomnia disorder and GAD implies the same genetic vulnerability for both conditions, and significant overlap in environmental influences (Lind et al., 2017). The latter finding suggests that insomnia disorder and GAD may share environmental influences, such as acute stressors, which may be valuable when considering the development and treatment of both disorders. As insomnia disorder and GAD often occur together and form a reciprocal relationship, a vital area for research is to examine treatment alternatives for patients with insomnia disorder co-morbid with GAD.

To our knowledge, only one previous treatment study has explored the efficacy of CBT-I for patients with insomnia disorder co-morbid with GAD. In a single-subject design, 10 patients were exposed to CBT-I and CBT for GAD in a sequenced manner (Belleville et al., 2016). The 10 participants were randomized to receive either CBT-I first (followed by CBT for GAD) or CBT for GAD first (followed by CBT-I). While CBT-I resulted in improvements in sleep quality and insomnia symptoms, there were no indications that CBT-I decreased worry and 
anxiety symptoms. CBT for GAD produced reductions in time worrying and sleep efficiency in most patients. CBT for GAD did not improve insomnia symptoms. There are other indications suggesting that worry does not hamper the efficacy of CBT-I. For example, one trial showed that low and high worriers achieved similar sleep outcomes after CBT-I (Hamoen et al., 2014).

The aim of the present study was to examine the efficacy of CBT-I among patients with insomnia disorder co-morbid with GAD. The investigation was based on theoretical notions as well as on a limited treatment research focusing on insomnia patients with co-morbid GAD. Based on the empirical literature, we reasoned that reduced insomnia symptoms could be expected to result in decreased anxiety and worry (e.g. Thielsch et al., 2015). One theoretical model, the Triple-R model (Maurer et al., 2018), proposes that treatment elements in CBT-I could have a profound impact on cognitive processes, such as reducing unhelpful beliefs and worry. As a result, we reasoned that CBT-I could decrease core symptoms in GAD. Turning to the limited treatment research on insomnia co-morbid with GAD, we decided to use a CBT-I manual that has an enhanced focus on both behavioural and cognitive maintaining processes and that has been shown to outperform therapies with a narrower focus (Harvey et al., 2014). Also, the current study aimed to assess outcome domains not indexed in the former trial (Belleville et al., 2016), i.e. functional impairment and quality of life, as these are vitally affected in patients with insomnia disorder and GAD. Finally, we aimed to examine putative mechanisms for CBT-I, based on the importance of such a focus in the treatment research area on both insomnia disorder and GAD. In this study, the putative mechanisms were chosen based on prominent behavioural and cognitive models of insomnia disorder (Harvey, 2002; Schwartz and Carney, 2012). These models build on the notion that homeostatic sleep pressure, the regulation of the sleep-wake cycle through circadian rhythms, and cognitive processes maintain insomnia disorder. As a result, behavioural (e.g. bedtime variability and time in bed) and cognitive processes (e.g. unhelpful beliefs and safety behaviours) were assessed in the current study.

Due to the limited extant research base, an open trial design was chosen. The efficacy of CBT-I was evaluated primarily on insomnia-related outcomes but also on anxiety, worry, depression, functional impairment, quality of life and adverse events. Also, measures indexing putative mechanisms for CBT-I were added. Based on theoretical notions, we hypothesized that, following CBT-I, symptoms of insomnia, anxiety and worry would be reduced, and that insomnia-related putative mechanisms would be reversed. For the remaining outcomes, the analyses were conducted mainly from an exploratory viewpoint due to limited prior evidence.

\section{Method}

\section{Overview of the study}

Patients were screened in three steps (screening questions, telephone interview and sleep diary) and then treated with CBT-I for 10 weeks. The patients were assessed at pre-treatment, bi-weekly during treatment, at post-treatment, and at 6-month follow-up. The study was reviewed and approved by the Regional Ethical Board in Stockholm, Sweden (reference number 2016/856-31). Based on recommendations by Whitehead and colleagues (2016), the aim was to recruit at least 15 participants to the study, with an estimated power of $90 \%$ and an expected medium difference between CBT-I and an active control condition for the primary outcome in a later trial.

\section{Recruitment, inclusion and exclusion criteria, and flow of participants}

A summary of the flow of participants through the study is presented in Fig. 1. The inclusion and exclusion criteria used during screening in three phases (i.e. web-administered screening questions, telephone interview, and sleep diary) were based on expert recommendation for a 


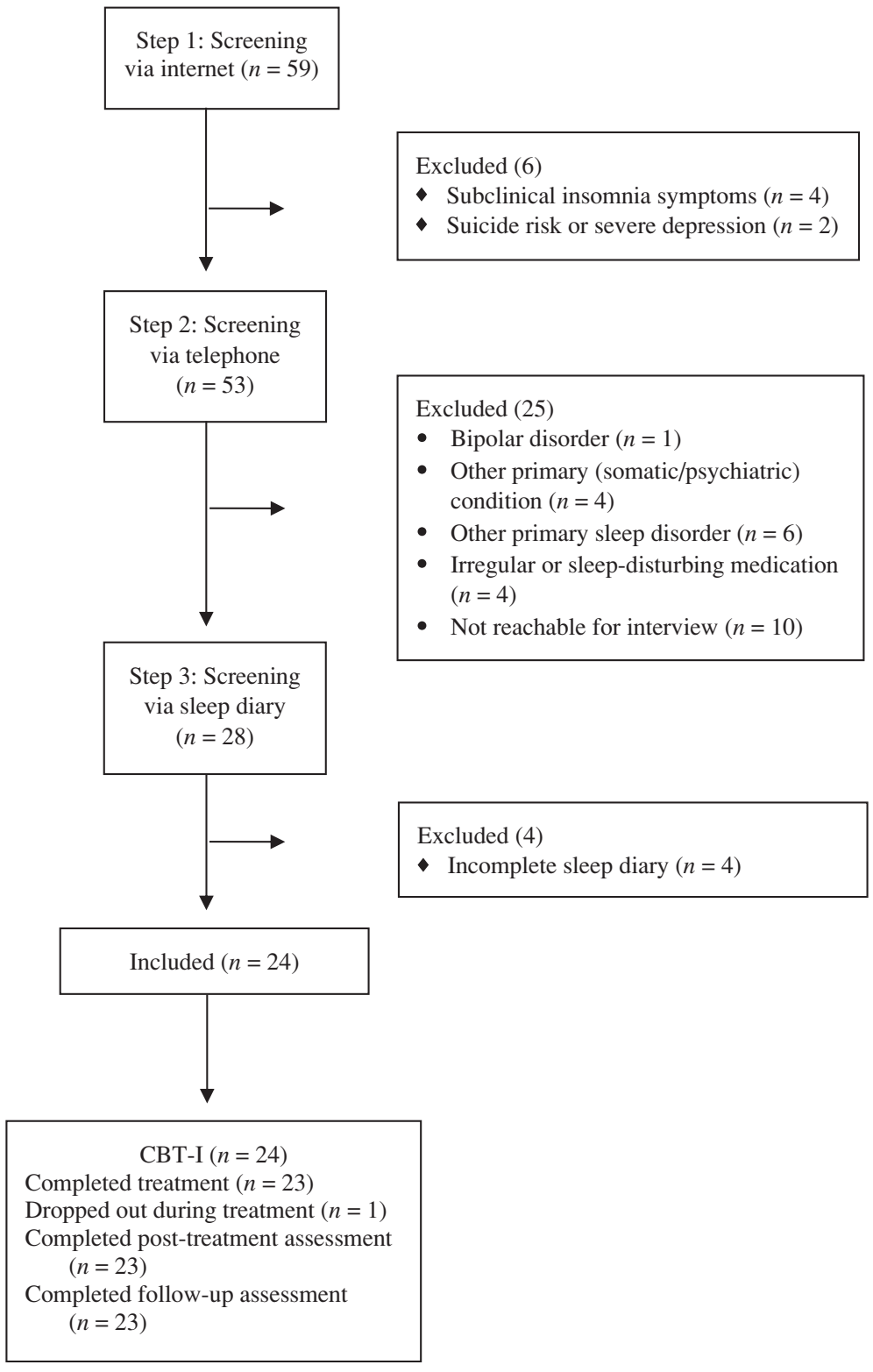

Figure 1. Flow diagram of study participants from screening to follow-up assessment.

standard research assessment of insomnia disorder and The Diagnostic and Statistical Manual of Mental Disorders-5 (American Psychiatric Association, 2013; Buysse et al., 2006; Edinger et al., 2004; Lichstein et al., 2003). Potential applicants were recruited via a newspaper advertisement in Stockholm (Sweden) and invited to receive information on a study-specific web platform and via telephone with one of the study authors. Applicants were then asked to sign a consent form and answer screening questions on the web platform. During the first stage of screening, applicants reported their sociodemographic parameters (age, gender, civil status, occupational status, educational level and country of birth), insomnia [the Insomnia Severity Index (ISI); Bastien et al., 2001], frequency and duration of insomnia symptoms, worry [the Penn State 
Worry Questionnaire (PSWQ); Meyer et al., 1990], depressive symptoms [the Patient Health Questionnaire (PHQ-9); Kroenke et al., 2001], and suicide risk (item 9 in the PHQ-9). Those who were: (1) 18 years or older, (2) exceeding the ISI cut-off at 10 points (Morin et al., 2011), (3) reporting clinical nighttime symptoms (i.e. 2 points or more on at least one of the three initial ISI questions), (4) stating clinical daytime symptoms (i.e. 2 points or more on ISI item 5 and/or item 7), (5) reporting occurrence of sleeping difficulties three nights or more per week over at least 3 months despite adequate opportunities for sleep, (6) exceeding the PSWQ cut-off at 45 points (Behar et al., 2003; Startup and Erickson, 2006), and (7) willing to participate in therapy for 10 weeks were eligible for the second round of screening. Applicants were also asked about their depression symptoms severity and suicidal ideation. Those who were severely depressed (more than 20 points on the PHQ-9; Kroenke et al., 2001) and/or reporting high suicidal ideation (i.e. better off dead or hurting oneself) but without plans or intentions (1 point or more on item 9 in the PHQ-9) were excluded from the trial. Applicants reporting severe depression and/or high suicidal ideation were referred to psychiatric care.

During the second screening phase, applicants were contacted for a semi-structured telephone interview based on the Duke Structured Interview for Sleep Disorders (DSISD) (Edinger et al., 2011) and the Mini-International Neuropsychiatric Interview (MINI) (Sheehan et al., 1997) to assess sleep and mental disorders. Also, these interviews aimed to identify and exclude applicants if sleeping problems were due to evident environmental conditions (e.g. pregnancy), night or rotating shift work ( $>3$ shifts a week), at least moderate intake of alcohol ( $>2$ standard drinks a day; Ebrahim et al., 2013) or caffeine ( $>4$ beverages a day or $>2$ after $18.00 \mathrm{~h}$ ), or if participation in CBT-I had occurred within the past 5 years. Further criteria required that insomnia disorder and GAD were disabling and distressing conditions, that reported somatic conditions were stable and/or that the applicant was receiving treatment for the condition, and that insomnia disorder and GAD were still present despite treatment for any somatic or psychiatric co-morbidity if co-morbidities were present. For medication, the following criteria were used: (a) if sleep medication was reported, it had to be relatively stable for the last 3 months, (b) if selective serotonin reuptake inhibitor (SSRI) use was reported, the onset of the medication, or the last change of dosage, should be at least 3 months prior to the telephone interview, and (c) if applicants were regularly consuming sleep-disturbing medications (e.g. benzodiazepines, pain medicines, and anti-hypertensives), they were excluded. Further criteria for exclusion were applicants with a history of psychotic or bipolar disorders or other current primary sleep disorders than insomnia disorder (sleep apnoea, restless legs syndrome, periodic limb movement disorder, circadian rhythm disorder, and parasomnias).

During the third screening phase, applicants completed a 7-day sleep diary (for details, see 'Insomnia symptoms' section below). The inclusion criterion at the third phase was to report at least 3 days of sleep difficulties in the sleep diary, i.e. sleep initiation, sleep maintenance or waking up too early, defined as $30 \mathrm{~min}$ or more for each occasion.

\section{Measures and procedure}

The ISI was used as the primary measure, and the remaining clinical outcomes as secondary measures. The self-report scales were administered at pre-treatment, post-treatment and 6-months follow-up. One of the self-report instruments, the ISI, was also completed by the participants bi-weekly during treatment (end of weeks 2, 4, 6 and 8). The sleep diary variables were filled out on paper daily from pre-treatment to post-treatment. At pre-treatment, biweekly (weeks 2, 4, 6 and 8), and at post-treatment, the participants transferred their sleep diary data to the digital platform. The putative mechanisms measures were administered at pre-treatment, biweekly and post-treatment. Adverse events and treatment satisfaction were only completed at post-treatment. Diagnostic measures as well as treatment credibility and 
expectancy were assessed with the study therapists, via telephone at pre-treatment or the first treatment session. The remaining measures were administered through an online, secure platform. Three automatic email reminders were sent out if an assessment had not been completed.

\section{Diagnostic measures}

To assess the diagnostic criteria for insomnia disorder, the DSISD was used (Edinger et al., 2011). The DSISD is a semi-structured interview with acceptable reliability (inter-rater reliability of 0.59 for insomnia related to a mental disorder) and validity. To identify GAD, the MINI 6.0.0 was used. The MINI is a psychometrically sound semi-structured clinical interview with acceptable reliability and validity (Sheehan et al., 1997), although there are indications that the MINI has limitations regarding the assessment of GAD (Verhoeven et al., 2017). The structured interviews were conducted by a trained and supervised masters student at the end of her clinical training.

\section{Treatment credibility, expectancy, completion and satisfaction}

To assess treatment credibility and expectancy of CBT-I, the Credibility/Expectancy Questionnaire (CEQ) (Devilly and Borkovec, 2000) was administered. CEQ is a 6-item questionnaire with demonstrated acceptable psychometric characteristics $(\alpha=.86$ in this sample) and has indicated the ability to predict outcome. The number of attended sessions and completed homework assignments were assessed by the study therapists. To determine treatment satisfaction, the Client Satisfaction Questionnaire (CSQ-8) was used (Attkisson and Zwick, 1982).

\section{Insomnia symptoms}

The Insomnia Severity Index (ISI) was used to assess participants' overall perception of insomnia severity (Bastien et al., 2001; Morin et al., 2011). The 7-item questionnaire is rated on a 5-point scale (0-4) with a total score of $0-28$ and assesses both night- and daytime symptoms. The ISI was also used as a screening tool (employing the validated cut-off at 10 points; Morin et al., 2011) and to categorize the number of responders (achieving a change of 8 points or more) and remitters (final score below 8) (Morin et al., 2011). In the current sample, the internal consistency (Cronbach's alpha) was acceptable at $\alpha=.81$.

To assess night-time symptoms, a 7-day sleep diary was administered (Carney et al., 2012). The diary assessed bedtime, lights-out time, sleep onset latency (SOL), wake time after sleep onset (WASO), early morning awakening (EMA) and rise-time. From these measures, the online diary automatically calculated total sleep time (TST), time in bed (TIB) and sleep efficiency (SE). Outcome measures were calculated as weekly means of SOL, WASO, EMA and TST. The sleep diary is viewed as the gold standard subjective measure of sleep (Buysse et al., 2006).

\section{Anxiety, worry, functional impairment, depression and quality of life}

To measure anxiety, worry, functional impairment, depression and quality of life, five psychometrically validated self-report measures were used. In the current sample, the internal consistency (Cronbach's alpha) was between .78 and .93 for the five scales. The Generalized Anxiety Disorder Screener (GAD-7) was employed to assess anxiety-related symptoms (Spitzer et al., 2006). The seven items are rated on a 4-point scale ranging from 1 (not at all) to 4 (nearly every day); higher scores indicate elevated anxiety symptoms. In the current study, a cut-off at $\geq 10$ points on the GAD-7 was used to estimate probable GAD-diagnosis (Spitzer et al., 2006). The PSWQ was used as an index of worry (Meyer et al., 1990). The 16 items are 
rated from 1 (not at all typical of me) to 5 (very typical of me); higher scores reflect elevated worry. To estimate pathological versus non-pathological worry, a cut-off at 45 points was used in the current study (Behar et al., 2003; Startup and Erickson, 2006). The Work and Social Adjustment Scale (WSAS) was used as an index of functional impairment (Jansson-Fröjmark, 2014; Mundt et al., 2002). The five-item questionnaire is rated on a 9-point scale (0-8) with a total score of 0-40; higher scores indicate elevated functional impairment. The Patient Health Questionnaire (PHQ-9) was employed to determine depression symptoms (Kroenke et al., 2001). The nine items are rated on a 4-point scale $(0=$ not at all; $3=$ nearly every day $)$ corresponding to the DSM-IV criteria for depression. The Brunnsviken Brief Quality of Life (BBQ) was used to evaluate quality of life (Lindner et al., 2016). The BBQ consists of 12 items assessing quality of life in six areas of life; higher scores indicate elevated quality of life.

\section{Adverse events}

Adverse events were assessed based on a checklist of 14 somatic and psychological events used in prior research (Kyle et al., 2011). The participants were asked to rate if any of the 14 adverse events (e.g. bodily pain, dizziness, low mood, and fatigue/exhaustion) had occurred as a result of treatment.

\section{Putative mechanisms}

Eight putative mechanisms were assessed. In this sample, the internal consistency (Cronbach's alpha) was between .73 and .94 for the eight measures. Four validated self-report scales were used to assess cognitive mechanisms. First, the Anxiety and Preoccupation about Sleep Questionnaire-2 (APSQ-2) was used to determine insomnia-related worry (Jansson-Fröjmark and Sunnhed, 2019). The two items in the APSQ-2 are scored from 1 (strongly disagree) to 10 (strongly agree); a higher score indicates elevated insomnia-related worry. Second, the Dysfunctional Beliefs about Sleep scale (DBAS-16) was employed to assess unhelpful beliefs about sleep (Morin et al., 2007). The response alternatives for the 16 DBAS items are from 0 (strongly disagree) to 10 (strongly agree); a higher score is suggestive of stronger unhelpful beliefs. Third, the Sleep Associated Monitoring Index-8 (SAMI-8) was used to determine attention for and monitoring of sleep-related threat (Jansson-Fröjmark and Sunnhed, 2019). The eight items in the SAMI-8 are scored from 1 (not at all) to 5 (all the time) with a total score range from 8 to 40; a higher score indicates elevated attention and monitoring. Fourth, the Sleep-Related Behaviours Questionnaire (SRBQ) was employed to assess insomnia-related safety behaviors (Ree and Harvey, 2004). The response alternatives for the 32 items are from 0 (almost never) to 4 (almost always); a higher score suggests elevated safety behaviours. To assess behavioural mechanisms, three items were calculated from the daily sleep diaries: bedtime variability, rise-time variability, and time in bed. Bedtime variability and rise-time variability were operationalized as the within-subject standard deviation for each participant (Edinger et al., 2001; Edinger et al., 2009). The two variability items were calculated by estimating the standard deviation across one week for each assessment point. Time in bed was estimated by calculating the time period between bedtime and rise-time for each night during a week (Edinger et al., 2001; Edinger et al., 2007; Edinger et al., 2009; Krystal and Edinger, 2010; Lichstein et al., 2001; Rybarczyk et al., 2002; Rybarczyk et al., 2005). The daily calculations for time in bed were then averaged across the week.

\section{CBT-I}

Cognitive Behavior Therapy for Insomnia (CBT-I) consisted of 10 weekly individual face-to-face therapy sessions lasting 60-75 minutes. The therapy followed a manual (Harvey et al., 2014). The number of weeks was determined based on previous research (Harvey et al., 2007; Harvey et al., 
2014). All sessions followed a structured agenda including (a) review of homework assignments, e.g. sleep diaries, (b) compliance issues and problem-solving centering around homework assignments, (c) rationale, skills training, and implementation of treatment components, and (d) homework assignments. The treatment was administered by two licensed clinical psychologists who had received training and supervision. To ensure treatment implementation, treatment delivery, receipt and enactment were monitored across all sessions, e.g. through documentation of all sessions by the therapist, patient hand-outs and using homework worksheets (Lichstein et al., 1994).

CBT-I contained a combination of a behaviour therapy (BT) and cognitive therapy (CT) approach. As in a previous trial on insomnia disorder (Harvey et al., 2014), case formulation (Harvey, 2005) was used to assess likely maintaining factors for each patient to determine the relative time and ordering of treatment components. The formulation was guided by the symptoms that were present and the patients' responses to questions, sleep diaries and questionnaires during the first sessions. While the first session was more generic [presenting the CBT approach, psychoeducation, introducing the $3 \mathrm{P}$ Model of Insomnia (Spielman et al., 1987a), keeping a sleep diary, and setting treatment goals], the remaining sessions were more devoted to implementing treatment components.

In the current study, three BT components were used across patients. First, sleep restriction (Spielman et al., 1987b) was implemented when the patient displayed excessive time in bed and low sleep efficiency. Sleep diaries were used to prescribe a sleep window for the coming week, which was then reviewed the following week. The sleep window was then increased or decreased depending on the sleep efficiency percentage (below or above $85 \%$ ). The second BT intervention, stimulus control (Bootzin, 1972), was used when there were indications that the patient engaged in sleep-incompatible behaviours within the bedroom environment, e.g. going to bed without signs of sleepiness, tossing and turning in bed, and displaying variability in rise-times. Third, sleep hygiene procedures were implemented when there were signs that the patient had bedroom circumstances and/or lifestyle factors perpetuating insomnia disorder, e.g. light exposure in bed and consuming caffeinated beverages in the hours before bedtime.

Building on a cognitive model of insomnia disorder (Harvey, 2002), treatment components were used to influence the proposed cognitive maintaining mechanisms: (a) unhelpful beliefs about sleep (Morin et al., 2002), (b) sleep-related or sleep-interfering worry (Tang and Harvey, 2004), (c) attentional bias and monitoring for sleep-related threat (Semler and Harvey, 2004), and (d) misperception of sleep (Harvey and Tang, 2012). Two core CT strategies were used to reverse the cognitive maintaining mechanisms: verbal, Socratic challenging (e.g. challenging an unhelpful, sleep-related belief or discussing the disadvantages of monitoring for internal or external threats) and behavioural experiments. As in a previous trial (Harvey et al., 2014), a minimum of four experiments was used for each patient: a monitoring/attentional bias experiment, the sleep survey experiment, the energy-generating experiment, and the fear of poor sleep experiment (Harvey et al., 2007). Following the cognitive model of insomnia disorder, attention and homework assignments were split between reversing the cognitive maintaining mechanisms during the daytime and the night-time (Harvey, 2002).

\section{Statistical analysis}

Treatment effects were examined using intention-to-treat linear mixed models. These models were used because, in the analysis of longitudinal data, repeated observations for the same individual are correlated, which violates the assumption of independence necessary for more traditional, repeated measure analysis and leads to bias in regression parameters. Furthermore, mixed-effect models can accommodate missing data by using all available data and the integration of time-varying factors, which are issues in the present study. Estimated parameters were obtained using a mixed-models approach employing a compound symmetry covariance 
Table 1. Descriptive statistics of the 24 study participants

\begin{tabular}{llc}
\hline & & Mean (SD) or \% (n) \\
\hline Age (years) & & $46.7(13.6)$ \\
Gender (female) & & $70.8 \%(17)$ \\
Civil status & Married, co-habiting with partner or having partner & $62.5 \%(15)$ \\
& Single & $37.5 \%(9)$ \\
Education (highest) & High school or university & $79.2 \%(19)$ \\
& Compulsory school & $20.8 \%(5)$ \\
Occupational status & Employed & $70.8 \%(17)$ \\
& Student & $12.5 \%(3)$ \\
Insomnia duration (years) & Retired or sick leave & $16.7 \%(4)$ \\
GAD duration (years) & & $10.7(9.8)$ \\
Somatic condition & & $9.3(7.7)$ \\
Psychiatric condition & & $25.0 \%(6)$ \\
Sleep medication & & $29.2 \%(7)$ \\
Other medication & & \\
\hline
\end{tabular}

${ }^{a}$ Headache (5 patients), respiratory diseases (2), heart diseases (1), gastric diseases (1) and chronic pain (1).

bSocial anxiety disorder (4 patients), major depression (3) and panic disorder (1).

${ }^{\mathrm{c}}$ For heart diseases (5 patients), anti-depressants (4), headache (3), tranquilizers (3), for asthma (2), allergy (1), and gastric issues (1).

structure because it provided the best model in an information criteria comparison. An alpha level of .05 was used as the criterion for statistical significance.

Estimated marginal means and standard deviations from mixed models were used to calculate effect sizes (ES). Within-group effect sizes (Cohen's $d$ ) were calculated [(pretreatment minus post-treatment or 6-month follow-up)/pooled standard deviation] to gain an impression of the magnitude of improvement associated with CBT-I. A threefold classification of effect sizes has been proposed (Cohen, 1988): small (0.20-0.49), medium (0.50-0.79) and large (0.80 and above). The same effect size analyses were conducted using observed data and the results were nearly identical and did not change our interpretation (data not shown).

\section{Results}

\section{Participant characteristics}

In total, 24 participants diagnosed with insomnia disorder and GAD were included in the study. A description of the participants is presented in Table 1.

\section{Treatment credibility, expectancy, completion and satisfaction}

According to the CEQ completed at session 1, treatment credibility [mean $=19.3(S D=3.7)$ ] and treatment expectancy $[$ mean $=18.3(S D=4.6)]$ were high. Session attendance for all 24 patients was on average at 9.1 sessions $(S D=2.4)$. One participant dropped out of the study before commencing CBT-I, and four patients attended less than the planned 10 sessions ( 8 sessions: $n=3,9$ sessions: $n=1$ ) due to time constraints and/or illness. Homework completion was, on average for all 24 patients, $88.2 \%(S D=12.9)$. According to the CSQ completed at posttreatment, treatment satisfaction was high $[$ mean $=25.7(S D=5.1)]$.

\section{Effects of CBT-I on clinical outcomes}

The descriptive statistics for the clinical outcomes are displayed in Table 2. First, analyses were executed to explore changes in the ISI. Mixed-effect models showed a significant effect of time on the ISI across the seven assessment points $(F=35.49, p<.001)$, with significant change occurring from pre-treatment to week 6 . The within-group effect sizes were large $(d=2.5-2.8)$. As can be 
Table 2. Estimated marginal means, standard deviations and effect sizes for the clinical outcomes - from pre-treatment to the 6-month assessment

\begin{tabular}{|c|c|c|c|c|c|c|c|c|c|}
\hline & Pre & Week 2 & Week 4 & Week 6 & Week 8 & Post & FU & $d$ & \\
\hline & Mean $(S D)$ & Mean $(S D)$ & Mean $(S D)$ & Mean $(S D)$ & Mean (SD) & Mean $(S D)$ & Mean $(S D)$ & Pre-Post & Pre-FU \\
\hline ISI & $19.9(3.9)$ & $13.8(3.9)$ & $12.6(4.6)$ & $11.5(5.4)$ & $10.3(4.0)$ & $8.4(4.4)$ & $9.8(4.2)$ & 2.75 & 2.48 \\
\hline $\mathrm{SOL}^{\mathrm{a}}$ & $56.9(44.5)$ & $38.0(20.8)$ & $28.2(14.4)$ & $24.1(13.1)$ & 25.9 (14.4) & $19.7(12.7)$ & - & 1.30 & 一 \\
\hline WASO $^{\mathrm{a}}$ & $46.0(35.6)$ & $33.5(23.3)$ & $27.0(15.7)$ & $24.6(19.1)$ & $23.8(14.4)$ & $15.8(11.0)$ & 一 & 1.30 & - \\
\hline $\mathrm{EMA}^{\mathrm{a}}$ & $38.9(32.6)$ & $25.3(29.3)$ & $18.4(22.0)$ & $15.5(22.5)$ & $9.8(14.4)$ & $15.9(24.6)$ & 一 & 0.80 & - \\
\hline $\mathrm{TST}^{\mathrm{a}}$ & $375.1(69.5)$ & $385.9(57.2)$ & 406.1 (73.4) & $416.1(59.8)$ & 421.9 (55.1) & $430.7(58.5)$ & - & 0.87 & - \\
\hline GAD-7 & $13.3(3.2)$ & - & - & - & - & $9.8(3.8)$ & $8.8(3.0)$ & 1.00 & 1.45 \\
\hline PSWQ & $64.9(9.4)$ & - & - & - & - & $58.4(10.9)$ & $54.3(9.8)$ & 0.64 & 1.10 \\
\hline PHQ-9 & $9.3(3.9)$ & & & & & $5.8(4.4)$ & $6.7(4.5)$ & 0.84 & 0.62 \\
\hline WSAS & $22.3(6.9)$ & - & - & - & - & $10.6(9.1)$ & $9.7(7.6)$ & 1.46 & 1.74 \\
\hline BBQ & $43.8(19.1)$ & - & - & - & - & $53.3(22.9)$ & $55.2(24.1)$ & 0.45 & 0.53 \\
\hline
\end{tabular}

BBQ, Brunnsviken Brief Quality of Life; $d$, Cohen's d; EMA, early morning awakening; FU, 6-month follow-up; GAD-7, Generalized Anxiety Disorder Screener-7; ISI, Insomnia Severity Index; PHQ-9, Patient Health Questionnaire-9; Pre, pre-treatment; Post, post-treatment; PSWQ, Penn State Worry Questionnaire; SOL, sleep onset latency; TST, total sleep time; WASO, wake after sleep onset; WSAS, Work and Social Adjustment Scale. ${ }^{a}$ Expressed in minutes.

Figure 2. Response and remission according to ISI cut-offs. Response: improvement of 8 points or more on the ISI from pre-treatment to post-treatment or follow-up; remission: score of less than 8 points on the ISI at posttreatment or follow-up.

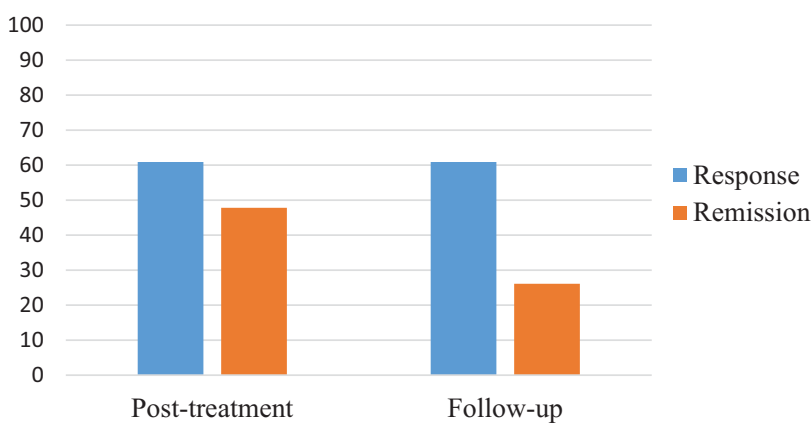

seen in Fig. 2, 60.9\% of the participants were treatment responders at both post-treatment and follow-up when using the ISI cut-offs. Also, $47.8 \%$ of the patients at post-treatment and $26.1 \%$ at follow-up met the criterion for remission.

Analyses on the four sleep diary outcomes were then executed. Mixed-effect models showed a significant effect of time on SOL $(F=13.53, p<.001)$, WASO $(F=10.09, p<.001)$, EMA $(F=7.31, p<.001)$ and TST $(F=9.38, p<.001)$. The significant changes emerged between pre-treatment and week 6 for SOL, WASO and TST, and from pre-treatment to week 2 for EMA. The within-group effect sizes on the four sleep diary parameters were large $(d=0.8-1.3)$.

The effects of CBT-I on the additional self-report measures (GAD-7, PSWQ, PHQ-9, WSAS and $\mathrm{BBQ}$ ) were then analysed. The analyses showed a significant effect of time on GAD-7 $(F=22.34, p<.001)$, PSWQ $(F=16.80, p<.001)$, PHQ-9 $(F=7.79, p=.001)$, WSAS $(F=49.16, p<.001)$ and BBQ $(F=7.84, p=.001)$. The significant changes occurred from pre- to post-treatment for GAD-7, PHQ-9, WSAS and BBQ, and from pre-treatment to follow-up for PSWQ. The within-group effect sizes on the four self-report scales were between medium and large $(d=0.5-1.7)$. Using the two GAD outcomes (PSWQ and GAD-7) to estimate the percentage of patients falling below validated cut-offs following CBT-I, 13.0\% scored below the PSWQ cut-off of 45 points at post-treatment and $26.1 \%$ at follow-up, and $33.3 \%$ had a total score below 10 points on the GAD-7 at post-treatment and $61.9 \%$ at follow-up. 
Table 3. Estimated marginal means, standard deviations and effect sizes for the putative mechanisms - from pretreatment to the post-treatment assessment

\begin{tabular}{|c|c|c|c|c|c|c|c|}
\hline & Pre & Week 2 & Week 4 & Week 6 & Week 8 & Post & $d$ \\
\hline & Mean $(S D)$ & Mean $(S D)$ & Mean $(S D)$ & Mean $(S D)$ & Mean $(S D)$ & Mean $(S D)$ & Pre-Post \\
\hline APSQ-2 & $17.3(3.1)$ & $13.9(5.4)$ & $11.8(5.0)$ & $11.4(5.6)$ & $10.9(5.8)$ & $10.3(5.9)$ & 1.56 \\
\hline DBAS-16 & $102.4(19.0)$ & $91.3(23.7)$ & $86.4(24.9)$ & $79.2(24.2)$ & $74.8(28.5)$ & $77.5(28.6)$ & 1.05 \\
\hline SAMI-8 & $26.1(4.4)$ & $24.2(5.4)$ & $23.4(5.4)$ & $21.9(6.3)$ & $21.4(6.8)$ & $20.7(6.6)$ & 0.98 \\
\hline SRBQ & $88.5(10.9)$ & $80.9(19.2)$ & 81.9 (19.9) & $76.9(21.8)$ & $77.4(22.4)$ & $74.6(22.4)$ & 0.83 \\
\hline Bedtime variability (min) & $44(24)$ & $42(23)$ & $43(25)$ & $39(20)$ & $44(30)$ & $33(21)$ & 0.49 \\
\hline Rise-time variability (min) & $57(32)$ & $58(26)$ & $57(31)$ & $53(27)$ & $61(32)$ & $51(36)$ & 0.18 \\
\hline Time in bed (min) & $518(38)$ & $481(43)$ & $478(68)$ & $485(53)$ & $482(48)$ & $481(41)$ & 0.94 \\
\hline
\end{tabular}

APSQ-2, Anxiety and Preoccupation about Sleep Questionnaire, 2-item version; $d$, Cohen's $d$; DBAS-16, Dysfunctional Beliefs and Attitudes about Sleep scale, 16-item version; Pre, pre-treatment; Post, post-treatment; SAMI-8, Sleep Associated Monitoring Index, 8-item version; SRBQ, Sleep-Related Behaviours Questionnaire.

\section{Adverse events}

Of the 23 patients that started CBT-I, seven (30.4\%) of the participants reported an adverse event at post-treatment. The reported events were in descending order (with the number of reports in parentheses): fatigue/exhaustion (5), extreme sleepiness (4), reduced motivation/energy (2), low mood (1), and dizziness (1).

\section{Effects of CBT-I on putative mechanisms}

The descriptive statistics for the putative mechanisms are displayed in Table 3. Mixed-effect models showed a significant effect of time on the four cognitive mechanisms: APSQ-2 $(F=16.39, p<.001)$, DBAS-16 $(F=15.23, p<.001)$, SAMI-8 $(F=11.41, p<.001)$ and SRBQ $(F=14.35, p<.001)$. The significant changes emerged between pre-treatment and week 6 for DBAS-16, SAMI-8 and SRBQ, and from pre-treatment to week 4 for APSQ-2. The within-group effect sizes for the cognitive mechanisms were large $(d=0.8-1.6)$. Furthermore, mixed-effect models demonstrated a significant effect on one of the three behavioural mechanisms with a large within-group effect size: time in bed $(F=9.42, p<.001)$. The significant changes occurred from pre-treatment to week 2 for time in bed. The two remaining behavioural mechanisms showed a non-significant effect [bedtime variability $(F=1.99, p=.086)$ and risetime variability $(F=0.64, p=.642)]$.

\section{Discussion}

The purpose of the current study was to explore the efficacy of CBT-I among patients with insomnia disorder co-morbid with GAD. The main findings were that CBT-I resulted in significant, large improvements in insomnia symptoms as well as significant improvements on the secondary clinical outcomes with moderate to large effect sizes, including GAD symptoms. Several of the putative mechanisms were reversed in the expected direction during CBT-I.

The efficacy of CBT-I in this trial was primarily directed at exploring changes in insomnia symptoms. Regarding insomnia severity, CBT-I resulted in large improvements $(d=2.5-2.8)$. Based on ISI cut-offs, approximately $61 \%$ of the participants responded to treatment and $48 \%$ remitted at post-treatment. Compared with a previous study using the same study protocol (but not specifically recruiting patients with co-morbid GAD), the response and remission rates were slightly lower (61 vs $67 \%$ and 48 vs 57\%) (Harvey et al., 2014). It is also important to underscore that only $26.1 \%$ of the patients reached insomnia remission at follow-up; this is markedly lower than the previous study mentioned above (56\%; Harvey et al., 2014). The reasons for a blunted response in rates of insomnia response and remission is not possible to 
tease out in the current study but might be due to GAD characteristics, such as excessive worry. Concerning night-time symptoms, CBT-I improved all four sleep diary parameters with large effect sizes. For the majority of insomnia symptoms, the significant changes occurred from pre-treatment to the week 6 assessment, which was followed by maintained improvements. Although CBT-I was delivered based on case conceptualization, resulting in slight variations across patients regarding which treatment components were used and in which order, all patients had been administered sleep restriction, stimulus control, Socratic questioning, and at least one behavioural experiment up to the sixth session. Based on a relatively solid evidence base for sleep restriction (Miller et al., 2014), it is likely that restricting time in bed was one important component for reducing insomnia symptoms in this trial.

Beyond reductions on insomnia symptoms, CBT-I resulted in significant improvements in anxiety, worry, depression, functional impairment and quality of life with medium to large effect sizes. The significant changes emerged between pre- and post-treatment for four of the domains with maintained results at follow-up. For worry, the significant change occurred across the whole study period, meaning that additional improvements were noted between post-treatment and follow-up; this continued reduction after CBT-I completion could not be observed on any other outcome at follow-up. Albeit not significant, the effect size for GAD-7 also suggested a continued improvement after CBT-I. In relation to the findings on anxiety and worry, it should be emphasized that the responses were limited using cut-offs; $33.3-61.9 \%$ of the patients scored below on the GAD-7 and 13.0-26.1\% on the PSWQ. Compared with the previous trial on insomnia disorder and GAD (Belleville et al., 2016), the larger effects on GAD symptoms might hypothetically be due to a strengthened cognitive therapy format of CBT-I in the current study. In particular, an apparent difference across studies is that the current study's version of CBT-I relied heavily on the main component in cognitive therapy, namely behaviuoral experiments. Such experiments build on a Socratic approach that relies on the patient drawing own conclusions with marked benefits, such as being rated as more helpful, supportive and engaging (Heiniger et al., 2018). Behavioural experiments also have the potential to be generalizable to other clinical presentations and problems. Although identical measures were not used across studies (Belleville et al., 2016), insomnia and GAD symptoms appear very similar (e.g. for ISI and PSWQ); pre-treatment symptom severity is thus not a likely explanation.

While the improvements on insomnia symptoms were expected, the large and continued reductions on worry and anxiety symptoms were not anticipated. Hypothetically, we present two explanations for the large improvements in worry and anxiety. First, the CBT-I components in this trial might be beneficial in decreasing GAD symptoms. For example, sleep restriction is likely to result in high sleep pressure and associated fatigue/exhaustion and reduced energy at the start of CBT-I (Kyle et al., 2011), which might reduce opportunities for worrying. Also, skills training on the cognitive components in CBT-I might generalize from insomnia to worry, through testing of worries concerning poor sleep and daytime symptoms. Concerning treatment components, it should be noted that no evidence-based elements for GAD, such as relaxation training (Borkovec and Costello, 1993), were used in the current study's CBT-I. Second, improved sleep during CBT-I might be viewed as an establishing operation (Michael, 2000). If so, it is conceivable that enhanced sleep quality and quantity is a stimulus condition that influences alternative behavioural patterns, such as activation, distraction, and problem-solving. In turn, such new behaviours might undercut the tendency to worry.

Approximately one-third of the patients experienced an adverse event at post-treatment. Based on previous findings showing that sleep restriction therapy results in increased fatigue/exhaustion and extreme sleepiness as well as reduced motivation/energy (Kyle et al., 2011), the adverse events reported in this study are likely to be due, at least partly, to sleep restriction. All seven putative mechanisms were changed in the expected direction following CBT-I. However, significant changes were noted on five of the seven mechanisms with large effect sizes. Similar 
improvements have been observed in previous trials (Harvey et al., 2007; Schwartz and Carney, 2012). It should be noted that the design of the study precludes conclusions regarding the putative mechanisms as mechanisms of change (Kazdin, 2007); this would, for example, require statistical analyses investigating temporal relations between changes in mechanisms and subsequent improvements in symptoms.

The current study has several methodological limitations that should be kept in mind when interpreting the findings. First, the efficacy of CBT-I was evaluated without a comparison group; future research should include a control group (e.g. waitlist or CBT for GAD conditions) to control for threats to internal validity. Second, based on previous research showing that GAD patients display objective sleep disturbance (Monti and Monti, 2000), future treatment studies might include objective sleep assessment. Third, CBT-I was administered by two therapists in the present study, thereby precluding conclusions regarding the efficacy of CBT-I to other clinicians. Other methodological alterations in future research could include recruiting patients from health care settings, having multiple interviewers, and assessing GAD symptoms (i.e. anxiety and worry) at more time-points during CBT-I.

The current study provides preliminary evidence for the efficacy of CBT-I for patients with insomnia disorder co-morbid with GAD. Before firm conclusions can be made on the efficacy of CBT-I for GAD, there is a need for replication of the present study and research using more robust designs.

Data availability. The data that support the findings of this study are available on request from the corresponding author (M.J.F.). The data are not publicly available due to containing information that could compromise the privacy of research participants.

Acknowledgements. We would like to express our appreciation to the Centre for Psychotherapy Research and Education, Centre for Psychiatry Research, Karolinska Institute for supporting the execution of this study.

Financial support. This research received no specific grant from any funding agency, commercial or not-for-profit sectors.

Conflict of interest. Markus Jansson-Fröjmark and Kalle Jacobson have no conflicts of interest with respect to this publication.

Ethical statements. We as authors have abided by the Ethical Principles of Psychologists and Code of Conduct as set out by the APA. The study was reviewed and approved by the Regional Ethical Board in Stockholm, Sweden (reference number 2016/ 856-31).

\section{References}

American Psychiatric Association (2013). Diagnostic and Statistical Manual of Mental Disorders (DSM-5). American Psychiatric Publishing.

Attkisson, C. C., \& Zwick, R. (1982). The Client Satisfaction Questionnaire: psychometric properties and correlations with service utilization and psychotherapy outcome. Evaluation and Program Planning, 5, 233-237.

Bastien, C. H., Vallières, A., \& Morin, C. M. (2001). Validation of the Insomnia Severity Index as an outcome measure for insomnia research. Sleep Medicine, 2, 297-307.

Behar, E., Alcaine, O., Zuellig, A. R., \& Borkovec, T. (2003). Screening for generalized anxiety disorder using the Penn State Worry Questionnaire: a receiver operating characteristic analysis. Journal of Behavior Therapy and Experimental Psychiatry, 34, 25-43.

Bélanger, L., Morin, C. M., Langlois, F., \& Ladouceur, R. (2004). Insomnia and generalized anxiety disorder: effects of cognitive behavior therapy for gad on insomnia symptoms. Journal of Anxiety Disorders, 18, 561-571.

Belleville, G., Ivers, H., Belanger, L., Blais, F. C., \& Morin, C. M. (2016). Sequential treatment of comorbid insomnia and generalized anxiety disorder. Journal of Clinical Psychology, 72, 880-896. doi: 10.1002/jclp.22300

Bootzin, R. R. (1972). Stimulus control treatment for insomnia. Proceedings of the American Psychological Association, 7 , 395-396.

Borkovec, T., \& Costello, E. (1993). Efficacy of applied relaxation and cognitive-behavioral therapy in the treatment of generalized anxiety disorder. Journal of Consulting and Clinical Psychology, 61, 611.

Brenes, G. A., Miller, M. E., Stanley, M. A., Williamson, J. D., Knudson, M., \& McCall, W. V. (2009). Insomnia in older adults with generalized anxiety disorder. American Journal of Geriatric Psychiatry, 17, 465-472. 
Breslau, N., Roth, T., Rosenthal, L., \& Andreski, P. (1996). Sleep disturbance and psychiatric disorders: a longitudinal epidemiological study of young adults. Biological Psychiatry, 39, 411-418.

Buysse, D. J., Ancoli-Israel, S., Edinger, J. D., Lichstein, K. L., \& Morin, C. M. (2006). Recommendations for a standard research assessment of insomnia. Sleep, 29, 1155-1173.

Carney, C. E., Buysse, D. J., Ancoli-Israel, S., Edinger, J. D., Krystal, A. D., Lichstein, K. L., \& Morin, C. M. (2012). The consensus sleep diary: standardizing prospective sleep self-monitoring. Sleep, 35, 287-302.

Cohen, J. (1988). Statistical Power Analysis for the Behavioral Sciences (2nd edn). Hillsdale, NJ, USA: Erlbaum.

Devilly, G. J., \& Borkovec, T. D. (2000). Psychometric properties of the credibility/expectancy questionnaire. Journal of Behavior Therapy and Experimental Psychiatry, 31, 73-86.

Ebrahim, I. O., Shapiro, C. M., Williams, A. J., \& Fenwick, P. B. (2013). Alcohol and sleep I: effects on normal sleep. Alcoholism: Clinical and Experimental Research, 37, 539-549.

Edinger, J. D., Bonnet, M. H., Bootzin, R. R., Doghramji, K., Dorsey, C. M., Espie, C. A., ... \& Stepanski, E. J. (2004). Derivation of research diagnostic criteria for insomnia: report of an American Academy of Sleep Medicine Work Group. Sleep, 27, 1567-1596.

Edinger, J. D., Olsen, M. K., Stechuchak, K. M., Means, M. K., Lineberger, M. D., Kirby, A., \& Carney, C. E. (2009). Cognitive behavioral therapy for patients with primary insomnia or insomnia associated predominantly with mixed psychiatric disorders: a randomized clinical trial. Sleep, 32, 499-510.

Edinger, J. D., Wohlgemuth, W. K., Radtke, R. A., Coffman, C. J., \& Carney, C. E. (2007). Dose-Response effects of cognitive-behavioral insomnia therapy: a randomized clinical trial. Sleep, 30, 203-212.

Edinger, J. D., Wohlgemuth, W. K., Radtke, R. A., Marsh, G. R., \& Quillian, R. E. (2001). Cognitive behavioral therapy for treatment of chronic primary insomnia: a randomized controlled trial. JAMA, 285, 1856-1864.

Edinger, J. D., Wyatt, J. K., Stepanski, E. J., Olsen, M. K., Stechuchak, K. M., Carney, C. E., . . \& Means, M. K. (2011). Testing the reliability and validity of DSM-IV-TR and ICSD-2 insomnia diagnoses: results of a multitrait-multimethod analysis. Archives of General Psychiatry, 68, 992-1002.

Ferre Navarrete, F., Perez Paramo, M., Fermin Ordono, J., \& Lopez Gomez, V. (2017). Prevalence of insomnia and associated factors in outpatients with generalized anxiety disorder treated in psychiatric clinics. Behavioral Sleep Medicine, 15, 491-501. doi: 10.1080/15402002.2016.1163703

Ford, D. E., \& Kamerow, D. B. (1989). Epidemiologic study of sleep disturbances and psychiatric disorders: an opportunity for prevention? JAMA, 262, 1479-1484.

Gehrman, P., Seelig, A. D., Jacobson, I. G., Boyko, E. J., Hooper, T. I., Gackstetter, G. D., . . \& Team, M. C. S. (2013). Predeployment sleep duration and insomnia symptoms as risk factors for new-onset mental health disorders following military deployment. Sleep, 36, 1009-1018.

Geiger-Brown, J. M., Rogers, V. E., Liu, W., Ludeman, E. M., Downton, K. D., \& Diaz-Abad, M. (2015). Cognitive behavioral therapy in persons with comorbid insomnia: a meta-analysis. Sleep Medicine Reviews, 23, 54-67.

Hamoen, A. B., Redlich, E. M., \& de Weerd, A. W. (2014). Effectiveness of cognitive behavioral therapy for insomnia: influence of slight-to-moderate depressive symptom severity and worrying. Depression and Anxiety, 31, 662-668. doi: $10.1002 /$ da. 22258

Harvey, A. G. (2002). A cognitive model of insomnia. Behaviour Research and Therapy, 40, 869-893.

Harvey, A. G. (2005). A cognitive theory and therapy for chronic insomnia. Journal of Cognitive Psychotherapy, 19, 41-59.

Harvey, A. G., Bélanger, L., Talbot, L., Eidelman, P., Beaulieu-Bonneau, S., Fortier-Brochu, É., ... \& Soehner, A. M. (2014). Comparative efficacy of behavior therapy, cognitive therapy, and cognitive behavior therapy for chronic insomnia: a randomized controlled trial. Journal of Consulting and Clinical Psychology, 82, 670.

Harvey, A. G., Sharpley, A. L., Ree, M. J., Stinson, K., \& Clark, D. M. (2007). An open trial of cognitive therapy for chronic insomnia. Behaviour Research and Therapy, 45, 2491-2501.

Harvey, A. G., \& Tang, N. K. (2012). (Mis) perception of sleep in insomnia: a puzzle and a resolution. Psychological Bulletin, 138, 77.

Heiniger, L. E., Clark, G. I., \& Egan, S. J. (2018). Perceptions of Socratic and non-Socratic presentation of information in cognitive behaviour therapy. Journal of Behavior Therapy and Experimental Psychiatry, 58, 106-113.

Jansson-Fröjmark, M. (2014). The work and social adjustment scale as a measure of dysfunction in chronic insomnia: reliability and validity. Behavioural and Cognitive Psychotherapy, 42, 186-198.

Jansson-Fröjmark, M., \& Lindblom, K. (2008). A bidirectional relationship between anxiety and depression, and insomnia? A prospective study in the general population. Journal of Psychosomatic Research, 64, 443-449.

Jansson-Fröjmark, M., \& Norell-Clarke, A. (2016). Cognitive behavioural therapy for insomnia in psychiatric disorders. Current Sleep Medicine Reports, 2, 233-240.

Jansson-Fröjmark, M., \& Sunnhed, R. (2019). Psychometric properties of two brief versions of cognitive, insomnia-specific measures: the Anxiety and Preoccupation About Sleep Questionnaire and the Sleep-Associated Monitoring Index. Psychological Reports, 0033294119832980.

Johnson, E. O., Roth, T., \& Breslau, N. (2006). The association of insomnia with anxiety disorders and depression: exploration of the direction of risk. Journal of Psychiatric Research, 40, 700-708. 
Kazdin, A. E. (2007). Mediators and mechanisms of change in psychotherapy research. Annual Review of Clinical Psychology, $3,1-27$.

Kim, B.-S., Jeon, H. J., Hong, J. P., Bae, J. N., Lee, J.-Y., Chang, S. M., . . \& Cho, M. J. (2012). DSM-IV psychiatric comorbidity according to symptoms of insomnia: a nationwide sample of Korean adults. Social Psychiatry and Psychiatric Epidemiology, 47, 2019-2033.

Kroenke, K., Spitzer, R. L., \& Williams, J. B. (2001). The PHQ-9: validity of a brief depression severity measure. Journal of General Internal Medicine, 16, 606-613.

Krystal, A. D., \& Edinger, J. D. (2010). Sleep EEG predictors and correlates of the response to cognitive behavioral therapy for insomnia. Sleep, 33, 669-677.

Kyle, S. D., Morgan, K., Spiegelhalder, K., \& Espie, C. A. (2011). No pain, no gain: an exploratory within-subjects mixedmethods evaluation of the patient experience of sleep restriction therapy (SRT) for insomnia. Sleep Medicine, 12, 735-747. doi: 10.1016/j.sleep.2011.03.016

Lichstein, K., Durrence, H., Taylor, D., Bush, A., \& Riedel, B. (2003). Quantitative criteria for insomnia. Behaviour Research and Therapy, 41, 427-445.

Lichstein, K. L., Riedel, B. W., \& Grieve, R. (1994). Fair tests of clinical trials: a treatment implementation model. Advances in Behaviour Research and Therapy, 16, 1-29.

Lichstein, K. L., Riedel, B. W., Wilson, N. M., Lester, K. W., \& Aguillard, R. N. (2001). Relaxation and sleep compression for late-life insomnia: a placebo-controlled trial. Journal of Consulting and Clinical Psychology, 69, 227.

Lind, M. J., Hawn, S. E., Sheerin, C. M., Aggen, S. H., Kirkpatrick, R. M., Kendler, K. S., \& Amstadter, A. B. (2017). An examination of the etiologic overlap between the genetic and environmental influences on insomnia and common psychopathology. Depression and Anxiety, 34, 453-462. doi: 10.1002/da.22587

Lindner, P., Frykheden, O., Forsström, D., Andersson, E., Ljótsson, B., Hedman, E., ... \& Carlbring, P. (2016). The Brunnsviken Brief Quality of life scale (BBQ): development and psychometric evaluation. Cognitive Behaviour Therapy, 45, 182-195.

Maurer, L. F., Espie, C. A., \& Kyle, S. D. (2018). How does sleep restriction therapy for insomnia work? A systematic review of mechanistic evidence and the introduction of the Triple- $\mathrm{R}$ model. Sleep Medicine Reviews, 42, 127-138.

McCall, W. V., Blocker, J. N., D’Agostino Jr, R., Kimball, J., Boggs, N., Lasater, B., \& Rosenquist, P. B. (2010). Insomnia severity is an indicator of suicidal ideation during a depression clinical trial. Sleep Medicine, 11, 822-827.

Mellinger, G. D., Balter, M. B., \& Uhlenhuth, E. H. (1985). Insomnia and its treatment - prevalence and correlates. Archives of General Psychiatry, 42, 225-232.

Meyer, T. J., Miller, M. L., Metzger, R. L., \& Borkovec, T. D. (1990). Development and validation of the Penn State Worry Questionnaire. Behaviour Research and Therapy, 28, 487-495.

Michael, J. (2000). Implications and refinements of the establishing operation concept. Journal of Applied Behavior Analysis, 33, 401-410.

Miller, C. B., Espie, C. A., Epstein, D. R., Friedman, L., Morin, C. M., Pigeon, W. R., . . \& Kyle, S. D. (2014). The evidence base of sleep restriction therapy for treating insomnia disorder. Sleep Medicine Reviews, 18, 415-424.

Monti, J. M., \& Monti, D. (2000). Sleep disturbance in generalized anxiety disorder and its treatment. Sleep Medicine Reviews, 4, 263-276. doi: 10.1053/smrv.1999.0096

Morin, C., Blais, F., \& Savard, J. (2002). Are changes in beliefs and attitudes about sleep related to sleep improvements in the treatment of insomnia? Behaviour Research and Therapy, 40, 741-752.

Morin, C. M., Belleville, G., Bélanger, L., \& Ivers, H. (2011). The Insomnia Severity Index: psychometric indicators to detect insomnia cases and evaluate treatment response. Sleep, 34, 601-608.

Morin, C. M., Drake, C. L., Harvey, A. G., Krystal, A. D., Manber, R., Riemann, D., \& Spiegelhalder, K. (2015). Insomnia disorder. Nature Reviews Disease Primers, 1, 15026.

Morin, C. M., Vallières, A., \& Ivers, H. (2007). Dysfunctional beliefs and attitudes about sleep (DBAS): validation of a brief version (DBAS-16). Sleep, 30, 1547-1554.

Mundt, J. C., Marks, I. M., Shear, M. K., \& Greist, J. M. (2002). The Work and Social Adjustment Scale: a simple measure of impairment in functioning. British Journal of Psychiatry, 180, 461-464.

Neckelmann, D., Mykletun, A., \& Dahl, A. A. (2007). Chronic insomnia as a risk factor for developing anxiety and depression. Sleep, 30, 873-880.

Qaseem, A., Kansagara, D., Forciea, M. A., Cooke, M., \& Denberg, T. D. (2016). Management of chronic insomnia disorder in adults: a clinical practice guideline from the American College of Physicians. Annals of Internal Medicine, 165, 125-133.

Ree, M. J., \& Harvey, A. G. (2004). Investigating safety behaviours in insomnia: the development of the Sleep-Related Behaviours Questionnaire (SRBQ). Behaviour Change, 21, 26-36.

Riemann, D., Baglioni, C., Bassetti, C., Bjorvatn, B., Dolenc Groselj, L., Ellis, J. G., . . \& Gonçalves, M. (2017). European guideline for the diagnosis and treatment of insomnia. Journal of Sleep Research, 26, 675-700.

Rybarczyk, B., Lopez, M., Benson, R., Alsten, C., \& Stepanski, E. (2002). Efficacy of two behavioral treatment programs for comorbid geriatric insomnia. Psychology and Aging, 17, 288. 
Rybarczyk, B., Stepanski, E., Fogg, L., Lopez, M., Barry, P., \& Davis, A. (2005). A placebo-controlled test of cognitivebehavioral therapy for comorbid insomnia in older adults. Journal of Consulting and Clinical Psychology, 73, 1164.

Schwartz, D. R., \& Carney, C. E. (2012). Mediators of cognitive-behavioral therapy for insomnia: a review of randomized controlled trials and secondary analysis studies. Clinical Psychology Review, 32, 664-675.

Semler, C. N., \& Harvey, A. G. (2004). An investigation of monitoring for sleep-related threat in primary insomnia. Behaviour Research and Therapy, 42, 1403-1420.

Sheehan, D., Lecrubier, Y., Sheehan, K. H., Janavs, J., Weiller, E., Keskiner, A., . . \& Dunbar, G. (1997). The validity of the Mini International Neuropsychiatric Interview (MINI) according to the SCID-P and its reliability. European Psychiatry, 12, 232-241.

Sivertsen, B., Lallukka, T., Salo, P., Pallesen, S., Hysing, M., Krokstad, S., \& Øverland, S. (2014). Insomnia as a risk factor for ill health: results from the large population-based prospective HUNT Study in Norway. Journal of Sleep Research, 23, 124-132.

Spielman, A. J., Caruso, L. S., \& Glovinsky, P. B. (1987a). A behavioral perspective on insomnia treatment. Psychiatric Clinics of North America, 10, 541-553.

Spielman, A. J., Saskin, P., \& Thorpy, M. J. (1987b). Treatment of chronic insomnia by restriction of time in bed. Sleep, 10, $45-56$.

Spitzer, R. L., Kroenke, K., Williams, J. B., \& Löwe, B. (2006). A brief measure for assessing generalized anxiety disorder: the GAD-7. Archives of Internal Medicine, 166, 1092-1097.

Startup, H. M., \& Erickson, T. M. (2006). The Penn State Worry Questionnaire (PSWQ). Worry and its Psychological Disorders: Theory, Assessment and Treatment, 101-119.

Sunderajan, P., Gaynes, B. N., Wisniewski, S. R., Miyahara, S., Fava, M., Akingbala, F., .. \& Trivedi, M. H. (2010). Insomnia in patients with depression: a STAR ${ }^{\star} \mathrm{D}$ report. CNS Spectrums, 15, 394-406.

Sylvia, L. G., Dupuy, J. M., Ostacher, M. J., Cowperthwait, C. M., Hay, A. C., Sachs, G. S., . . \& Perlis, R. H. (2012). Sleep disturbance in euthymic bipolar patients. Journal of Psychopharmacology, 26, 1108-1112.

Tang, N. K., \& Harvey, A. G. (2004). Correcting distorted perception of sleep in insomnia: a novel behavioural experiment? Behaviour Research and Therapy, 42, 27-39.

Taylor, D. J., Lichstein, K. L., Durrence, H. H., Reidel, B. W., \& Bush, A. J. (2005). Epidemiology of insomnia, depression, and anxiety. Sleep, 28, 1457-1464.

Thielsch, C., Ehring, T., Nestler, S., Wolters, J., Kopei, I., Rist, F., . . \& Andor, T. (2015). Metacognitions, worry and sleep in everyday life: studying bidirectional pathways using Ecological Momentary Assessment in GAD patients. Journal of Anxiety Disorders, 33, 53-61. doi: 10.1016/j.janxdis.2015.04.007

Troxel, W. M., Kupfer, D. J., Reynolds, C. F., Frank, E., Thase, M., Miewald, J., \& Buysse, D. J. (2012). Insomnia and objectively measured sleep disturbances predict treatment outcome in depressed patients treated with psychotherapy or psychotherapy-pharmacotherapy combinations. Journal of Clinical Psychiatry, 73, 478.

Verhoeven, F. E. A., Swaab, L. S. M. A., Carlier, I. V. E., van Hemert, A. M., Zitman, F. G., Ruhe, H. G., . . \& Giltay, E. J. (2017). Agreement between clinical and MINI diagnoses in outpatients with mood and anxiety disorders. Journal of Affective Disorders, 221, 268-274.

Whitehead, A. L., Julious, S. A., Cooper, C. L., \& Campbell, M. J. (2016). Estimating the sample size for a pilot randomised trial to minimise the overall trial sample size for the external pilot and main trial for a continuous outcome variable. Statistical Methods in Medical Research, 25, 1057-1073.

Wu, J. Q., Appleman, E. R., Salazar, R. D., \& Ong, J. C. (2015). Cognitive behavioral therapy for insomnia comorbid with psychiatric and medical conditions: a meta-analysis. JAMA Internal Medicine, 175, 1461-1472.

Zachariae, R., Lyby, M. S., Ritterband, L. M., \& O’Toole, M. S. (2016). Efficacy of internet-delivered cognitive-behavioral therapy for insomnia - a systematic review and meta-analysis of randomized controlled trials. Sleep Medicine Reviews, 30, 1-10.

Cite this article: Jansson-Fröjmark M and Jacobson K (2021). Cognitive behavioural therapy for insomnia for patients with co-morbid generalized anxiety disorder: an open trial on clinical outcomes and putative mechanisms. Behavioural and Cognitive Psychotherapy 49, 540-555. https://doi.org/10.1017/S1352465821000023 